\title{
Morinda citrifolia and Its Active Principle Scopoletin Mitigate Protein Aggregation and Neuronal Apoptosis through Augmenting the DJ-1/Nrf2/ARE Signaling Pathway
}

\author{
Kishore Kumar S. Narasimhan, ${ }^{1,2}$ Deepthy Jayakumar, ${ }^{1}$ Prema Velusamy, ${ }^{1}$ \\ Ashokkumar Srinivasan, ${ }^{1}$ Thangarajeswari Mohan, ${ }^{1}$ Divya Bhavani Ravi, ${ }^{1}$ \\ Saraswathi Uthamaraman, ${ }^{1}$ Yogesh Kanna Sathyamoorthy, ${ }^{3}$ \\ Namakkal Soorappan Rajasekaran $\mathbb{D D}^{2}$ and Kalaiselvi Periandavan ${ }^{1}{ }^{1}$ \\ ${ }^{1}$ Department of Medical Biochemistry, Dr. ALM Post Graduate Institute for Basic Medical Sciences, University of Madras, \\ Chennai, India \\ ${ }^{2}$ Cardiac Aging \& Redox Signaling Laboratory, Division of Molecular \& Cellular Pathology, Department of Pathology, \\ University of Alabama, Birmingham, AL 35294, USA \\ ${ }^{3}$ Department of Anatomy, Dr. ALM Post Graduate Institute for Basic Medical Sciences, University of Madras, Chennai, India \\ Correspondence should be addressed to Namakkal Soorappan Rajasekaran; rajnsr@uabmc.edu \\ and Kalaiselvi Periandavan; pkalaiselvi2011@gmail.com
}

Received 1 October 2018; Revised 16 January 2019; Accepted 12 February 2019; Published 2 May 2019

Guest Editor: Luciana Scotti

Copyright (c) 2019 Kishore Kumar S. Narasimhan et al. This is an open access article distributed under the Creative Commons Attribution License, which permits unrestricted use, distribution, and reproduction in any medium, provided the original work is properly cited.

\begin{abstract}
Given the role of oxidative stress in PD pathogenesis and off-target side effects of currently available drugs, several natural phytochemicals seem to be promising in the management of PD. Here, we tested the hypothesis that scopoletin, an active principle obtained from Morinda citrifolia (MC), efficiently quenches oxidative stress through DJ-1/Nrf2 signaling and ameliorates rotenone-induced PD. Despite reducing oxidative stress, the administration of MC extract (MCE) has lessened protein aggregation as evident from decreased levels of nitrotyrosine and $\alpha$-synuclein. In vitro studies revealed that scopoletin lessened rotenone-induced apoptosis in SH-SY5Y cells through preventing oxidative injury. Particularly, scopoletin markedly upregulated DJ-1, which then promoted the nuclear translocation of $\mathrm{Nrf} 2$ and transactivation of antioxidant genes. Furthermore, we found that scopoletin prevents the nuclear exportation of Nrf2 by reducing the levels of Keap1 and thereby enhancing the neuronal defense system. Overall, our findings suggest that scopoletin acts through DJ-1-mediated Nrf2 signaling to protect the brain from rotenone-induced oxidative stress and PD. Thus, we postulate that scopoletin could be a potential drug to treat PD.
\end{abstract}

\section{Introduction}

Although the causal mechanisms of Parkinson's disease $(\mathrm{PD})$ remain elusive, excess production of reactive oxygen species (ROS), mitochondrial dysfunction, neuroinflammation, and environmental toxins are reported to promote the loss of dopaminergic neurons in PD [1]. Oxidative stress has been shown to induce misfolding, aggregation, and accumulation of such aggregates leading to the pathogenesis of many neurodegenerative diseases including PD [2]. Intracellular inclusions known as Lewy bodies (LBs) are regarded as a hallmark of common pathological manifestation in both familial and sporadic PD patients with $\alpha$-synuclein ( $\alpha$-Syn) serving as the main component of LB [3]. $\alpha$-Syn is natively unfolded and is prone to form fibrils during oxidative stress [4], indicating that redox signaling may play a significant role in the aggregation of $\alpha$-Syn. 
Previous studies have reported that the loss of antioxidant defense aggravates PD progression [5, 6]. A key example includes DJ-1/PARK7, a molecular chaperone known to regulate Keap1-Nrf2 signaling, which is the primary sensor for reactive electrophiles activating Nrf2 nuclear translocation and transactivation of the antioxidant response element (ARE) in a battery of cytoprotective genes facilitating protection from oxidative stress pathogenesis [7] including experimental models of PD [8]. Thus, pharmacological activation of the Nrf2 in the brain is likely to preserve neuronal health. Therefore, exploration for therapeutic compounds with lesser neurotoxic effects that activates Nrf2 signaling would be promising to treat PD.

In this context, identifying potential principles from medicinal plants would be ideal as plant extracts have been reported to have several therapeutic benefits, due to the synergistic effect of various natural ingredients [9]. However, such plant sources have not been in clinical practice or in global market due to the lack of scientific validation. Morinda citrifolia fruit extract (MCE) has potential antioxidant properties due to the presence of several active constituents (including scopoletin and quercetin) and protects skeletal muscle from apoptosis [10] and also prevents striatal degeneration [11] in experimental Parkinsonian rats. Here, we hypothesized that MCE and scopoletin prevent rotenoneinduced oxidative stress and apoptosis through the activation of DJ-1/Nrf2 signaling and investigated its neuroprotective effects using in vivo (Sprague-Dawley rats) and in vitro (SHSY5Y cells) models of PD.

\section{Materials and Methods}

2.1. Animals, Intranigral Rotenone Infusion, and Treatment. Adult male Sprague-Dawley rats were used in this study. All experiments were performed in accordance with the guidelines approved by the Institutional Animal Ethical Committee (IAEC No. 01/09/12). Rats were divided into five groups ( $n=10 / \mathrm{gp}$ ). Group I served as control, while groups II to $\mathrm{V}$ were subjected to stereotaxic surgery. Group II served as sham controls and groups III-V were stereotaxically infused with rotenone to induce Parkinsonism. Briefly, rats were anaesthetized with ketamine hydrochloride and xylazine ( $80 \mathrm{mg} / \mathrm{kg}$ and $10 \mathrm{mg} / \mathrm{kg}$; i.p.) and placed on a small animal stereotaxic frame (Stoelting, IL, USA). Rotenone dissolved in DMSO $(1 \mu \mathrm{g} / 1 \mu \mathrm{l})$ was infused into the right ventral tegmental area (VTA, anterior-posterior (AP): $5.0 \mathrm{~mm}$; laterally (L): $1.0 \mathrm{~mm}$; dorso-ventral (DV): $7.8 \mathrm{~mm}$ ) and into the right substantia nigra pars compacta $(\mathrm{SNPc}, \mathrm{AP}: 5.0 \mathrm{~mm}$; L: $2.0 \mathrm{~mm}$; DV: $8.0 \mathrm{~mm}$ ) at a flow rate of $0.2 \mu \mathrm{l} / \mathrm{min}$ using a Hamilton 26 -gauge needle $[10,11]$. The infusion needle was left in place for additional five minutes for complete diffusion of the drug. Sham controls were infused with DMSO and polyethylene glycol in the ratio of $1: 1$ during stereotaxic surgery. Two weeks postsurgery, rats in groups IV and V were treated with levodopa (LD, $100 \mathrm{mg} / \mathrm{kg}$ with $25 \mathrm{mg} / \mathrm{kg}$ benserazide [12]) and ethyl acetate extract of Morinda citrifolia fruit (MCE, $150 \mathrm{mg} / \mathrm{kg}$ body weight), respectively, for the next 30 days. To determine the efficiency of intranigral infusion of rotenone, animals were subjected to behavioural analysis [11].
2.2. In Vitro Studies Using SH-SY5Y Cells. SH-SY5Y cells were initially grown in 1:1 mixture of DMEM and F12K Medium supplemented with $10 \%$ fetal bovine serum $(v / v)$, $100 \mathrm{U} / \mathrm{ml}$ penicillin, and $100 \mu \mathrm{g} / \mathrm{ml}$ streptomycin in a $25 \mathrm{~cm}^{2}$ and $75 \mathrm{~cm}^{2}$ vented culture flasks. The cultures were incubated at $37^{\circ} \mathrm{C}$ in $5 \% \mathrm{CO}_{2} / 95 \%$ humidified air. When cells had reached $80-90 \%$ confluence in the flask, they were trypsinized and seeded onto 96-well plates or 6-well plates. Rotenone and scopoletin were dissolved in dimethyl sulfoxide (DMSO, final concentration of DMSO was $0.01 \%$ ). Rotenone $(500 \mathrm{nM})$ was used for $24 \mathrm{~h}$ to induce cell damage [13]. Preliminary studies were carried out with different concentrations $(1 \mu \mathrm{M}, 10 \mu \mathrm{M}, 30 \mu \mathrm{M}, 50 \mu \mathrm{M}$, and $100 \mu \mathrm{M})$ and different time intervals $(0 \mathrm{~h}, 1 \mathrm{~h}, 3 \mathrm{~h}$, and $6 \mathrm{~h})$ of scopoletin to fix the time of exposure and dosage using MTT assay and the optimal concentration was found to be $30 \mu \mathrm{M}$ pretreated for $3 \mathrm{~h}$ (Supplementary Figure 2). To investigate whether scopoletin protects cells from rotenone-induced cell death, cells were divided into three groups: control group, rotenone group (treated with rotenone for $24 \mathrm{~h}$ ), and scopoletin group (pretreated with $30 \mu \mathrm{M}$ scopoletin for $3 \mathrm{~h}$ followed by exposure to rotenone).

2.3. Analysis of Apoptotic Cells by Flow Cytometry. The different stages of apoptosis in control and treated SH-SY5Y cells were analyzed by flow cytometry using annexin V-FITC/PI double staining kit (Cayman Chemicals, Carlsbad, USA). After the incubation period, cells were washed with cold phosphate buffered saline (PBS), centrifuged twice at $1500 \mathrm{rpm}$ for $5 \mathrm{~min}$, and suspended in $500 \mu \mathrm{l}$ of binding buffer. FITC-labeled annexin V $(5 \mu \mathrm{l})$ and propidium iodide (PI, $5 \mu \mathrm{l}$ ) were added and incubated with the cells at room temperature for $15 \mathrm{~min}$. Apoptotic cells were measured using a FACSCalibur flow cytometer (Becton Dickinson, NJ, USA). Annexin V-positive, PI-negative cells were scored as early apoptotic cells. Cells that were positive for both annexin V and PI were considered as late apoptotic cells.

2.4. $m R N A$ Expression Studies by Reverse TranscriptionPolymerase Chain Reaction (RT-PCR). Total RNA was isolated from the striatal tissue using total RNA isolation reagent (TRIZOL, Invitrogen, Carlsbad, CA, USA). Oligonucleotide primer sequences (Supplementary Table 1) of the selected genes for reverse transcription-polymerase chain reaction (RT-PCR) were synthesized by Sigma-Aldrich (St. Louis, MO, USA) and Eurofins Genomics (India). The amplified products were separated by electrophoresis on 1-2\% agarose gel. Specificity was confirmed by the size of the amplified products with reference to $100 \mathrm{bp}$ DNA ladder (BioVision, USA) and the band intensities were quantified by Quantity One Software (Bio-Rad, USA).

2.5. Western Blotting. Striatal tissue lysates ( $50 \mu \mathrm{g}$ protein) and SH-SY5Y cell lysates were separated by SDS-PAGE on $10-12 \%$ polyacrylamide gels and transferred to polyvinylidene difluoride membranes. The membranes were incubated with specific primary antibodies and the antibodies used were DJ-1, Nrf2, phosphoS40-Nrf2 (Abcam 1:1000 dilution), Keap1, NQO1, Cullin3, PKC- $\delta$ (Pierce Antibodies, 
$1: 1000$ dilution), $\gamma$ GCLC, HO-1, nitrotyrosine (Santa Cruz Biotech, $1: 1000$ dilution), $\alpha$-synuclein, and iNOS (Cell Signaling Technology, 1:1000 dilution). To verify the uniformity of protein load and transfer efficiency across the test samples, membranes were reprobed with $\beta$-actin and Lamin B (Cell Signaling Technology, $1: 1000$ dilution). Immunoreactive bands were developed with Immobilon Western Chemiluminescent HRP substrate (Millipore Corporation, Billerica, USA) and visualized by using an enhanced chemiluminescence system (ChemiDoc, Bio-Rad, USA) and presented in comparison to $\beta$-actin/Lamin $\mathrm{B}$ expression.

2.6. Statistical Analysis. Data are presented as mean \pm standard error of mean (SEM) of the results obtained from the average of at least three to six independent experiments. Results were analyzed by one-way analysis of variance (ANOVA) using the SPSS software package for Windows (Version 20.0; SPSS Inc., Chicago, IL, USA) and $p$ values were determined using the Student-Newman-Keuls and least significant difference post hoc test. Differences among means were considered statistically significant when the $p$ value was less than 0.05 .

\section{Results}

3.1. Impact of MCE on Nigrostriatal Tyrosine Hydroxylase (TH) Immunoreactivity. Immunohistochemical localization of TH-positive neurons in the striatum as well as the substantia nigra pars compacta (SNPc) of control and experimental rats is presented in Figure 1(a). Histochemical analysis of SNPc along with the striatum makes it convenient to understand the efficacy of the MCE treatment. The striatum ipsilateral to the side of infusion showed significant loss of $\mathrm{TH}$ immunostaining. TH immunoreactivity of the ipsilateral striatum shows a remarkable refurbishment of dopaminergic neurons seen in MCE-administered rats. Rotenone-infused SNPc showed less number of TH-positive cells as compared to control animals. MCE treatment in these animals restored, to a great extent, the loss of these cells. While there was a $43 \%$ decrease in $\mathrm{TH}$-positive neurons in response to rotenone administration, MCE treatment curtailed this to $30 \%$ (Figure 1(b)).

\subsection{MCE Counteracts Rotenone-Induced Oxidative Stress in} Experimental PD Rats. Analyses of various oxidative stress markers indicated that MCE was an efficacious treatment to reduce oxidative stress in rotenone-induced $\mathrm{PD}$ rats. We first determined the levels of nitric oxide (NO) which was significantly increased $(p<0.05)$ in the striatum of rotenone-infused $\mathrm{PD}$ rats and this was blunted in response to MCE treatment. Next, quantification of lipid peroxidation (LPO) and protein carbonyls (PC) revealed that rotenone infusion increased the levels of these oxidative byproducts to 22 and $41 \%$, respectively, in relation to sham controls (Figure 1(c)). However, upon treating with MCE, LPO and PC content was significantly decreased.

3.3. MCE Protects from Rotenone-Induced Protein Aggregation. Considering the interrelationships between nitric oxide, oxidative stress, and protein aggregation [14], we further determined the impact of MCE on the levels of iNOS and nitrotyrosine as the former is involved in the synthesis of NO and the latter is a marker for protein aggregation. Rotenone-infused Parkinsonian rats showed a significant $(p<0.05)$ increase in the protein levels of iNOS and nitrotyrosine when compared with controls (Figure 1(d)). Treatment with MCE significantly ameliorated rotenone-mediated NO production through diminishing the levels of iNOS. As a result, we also found that MCE was particularly efficient in blocking the formation of nitrotyrosine adducts (i.e. $\alpha$-synuclein), which are the primary events in the process of protein aggregation that occurs in response to oxidative insults, such as those triggered by rotenone.

To further confirm the suppression of protein aggregation by MCE, we analyzed both the protein levels and the immunostaining for $\alpha$-synuclein ( $\alpha$-Syn), a pathological protein that is aggregated in PD. While immunostaining showed a significant increase in $\alpha$-Syn aggregation (Figures 2(a) and 2(b)) in the striatum of rotenone-induced rats which correlates with the increase in the nitrotyrosine levels, MCE treatment abolished these changes and significantly reduced the aggregation of $\alpha$-Syn suggesting that oxidative stress promotes the aggregation of $\alpha$-Syn. Moreover, rotenone-infused Parkinsonian rats exhibited a significant $(p<0.05)$ increment in the protein levels of $\alpha$-Syn by $79 \%$ (Figure 2(c)), which further aggravated the aggregation of $\alpha$ Syn in these rats.

3.4. MCE Prevents Rotenone-Induced Oxidative Stress by Augmenting the Antioxidant Defense. We postulated that the antioxidative potential of MCE might be associated with diminished oxidative stress. A significant decline $(p<0.05)$ in the activities of antioxidant enzymes, namely, superoxide dismutase (SOD), catalase, glutathione peroxidase (GPx), glutathione reductase (GR), and glutathione-S-Transferase (GST), was observed (Table 1(a)) in the rotenone-induced Parkinsonian rats when compared with controls. However, upon supplementing with MCE, a significant augmentation in the activities of SOD, CAT, and GST was observed with a maximum improvement in the SOD activity (36\%). However, there were no significant changes in the activities of other enzymatic antioxidants GPx, GR. In addition, the levels of reduced glutathione (GSH), a vital ubiquitous antioxidant thiol, decreased in response to rotenoneinduced oxidative stress. MCE supplementation significantly increased the levels of GSH back to near normal when compared with the rotenone-infused rats. Because the Nrf2 pathway transcriptionally activates glutathione-biosynthesizing enzymes, we next assessed whether GSH changes were mechanistically linked to Nrf2 signaling in the MCEtreated animals.

3.5. MCE Induces Nrf2/ARE Pathway and Suppresses Rotenone-Induced Oxidative Stress. The downregulation of the Nrf2/ARE pathway exacerbates oxidative stress which potentiates dopaminergic degeneration and pathogenesis of PD [15]. As we noticed a significant alteration in cellular redox status, elevated $\alpha$-Syn expression, and aggregation in the current study, we further analyzed the levels of Nrf2 


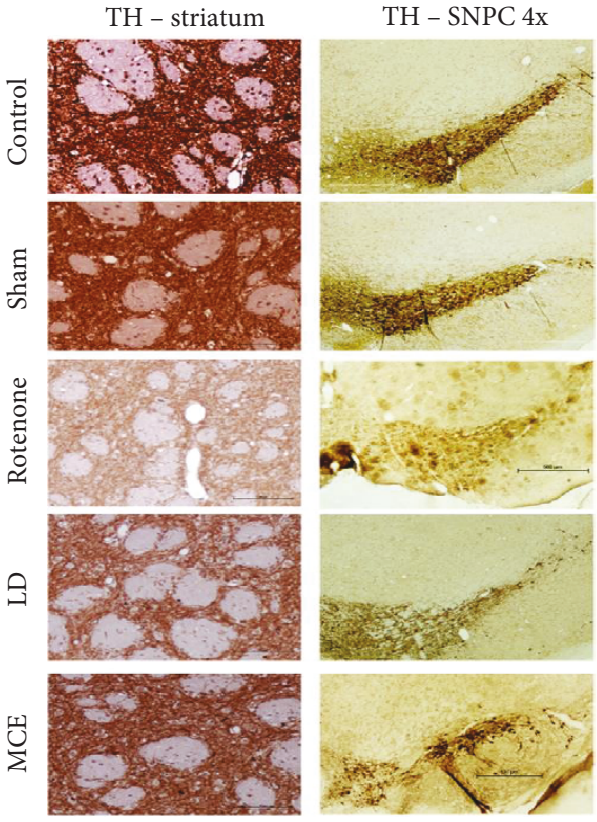

(a)
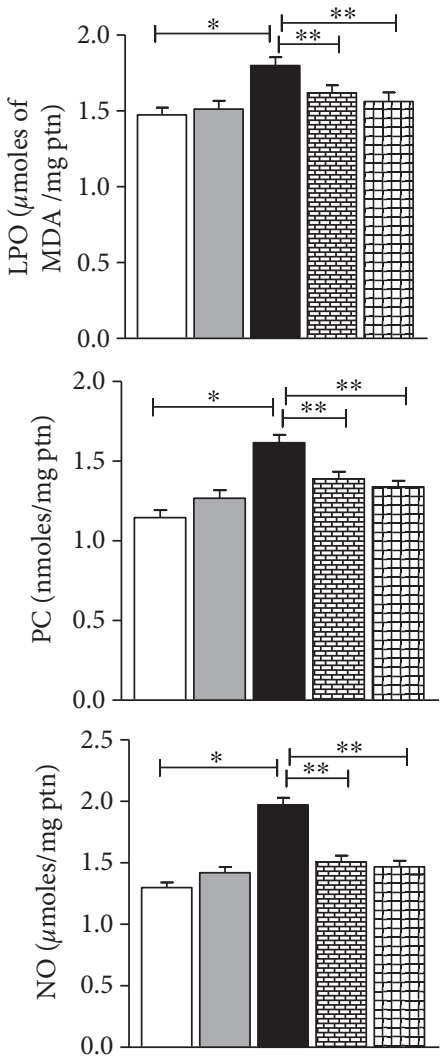

(c)

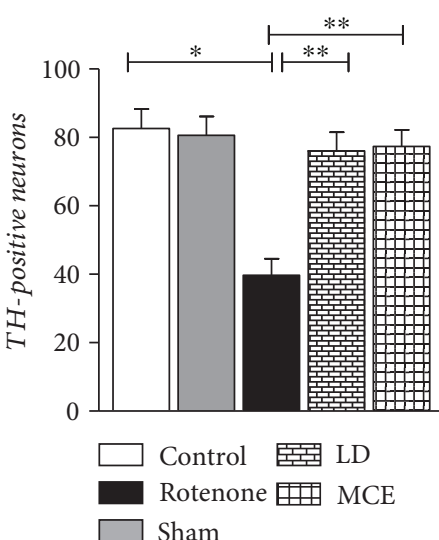

(b)

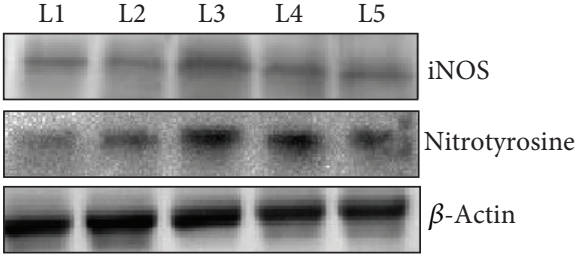

L1: Control; L2: Sham; L3: Rot Ind; L4: Rot+LD; L5: Rot+MCE

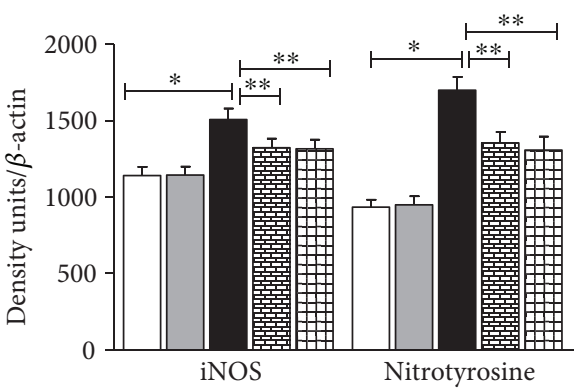

(d)

FIgure 1: (a) Photographic representation of the TH-positive neurons in the striatum and in SNPc. (b) Quantification of relative intensity of $\mathrm{TH}$ staining in the striatum using the densitometry protocol through ImageJ was performed to substantiate the potentials of salvaging activity of MCE. (c) Impact of MCE on rotenone-induced oxidative stress: values are expressed as for six animals in each group. (d) Immunoblot analysis of iNOS and nitrotyrosine (i.e. $\alpha$-synuclein) and representative densitometry quantification. Statistical significance $(p<0.05)$ was calculated by Student-Newman-Keuls and least significant difference post hoc test, where ${ }^{*}$ represents control vs. other groups, ${ }^{* *}$ represents rotenone vs. $\mathrm{LD}, \mathrm{MCE}$. 

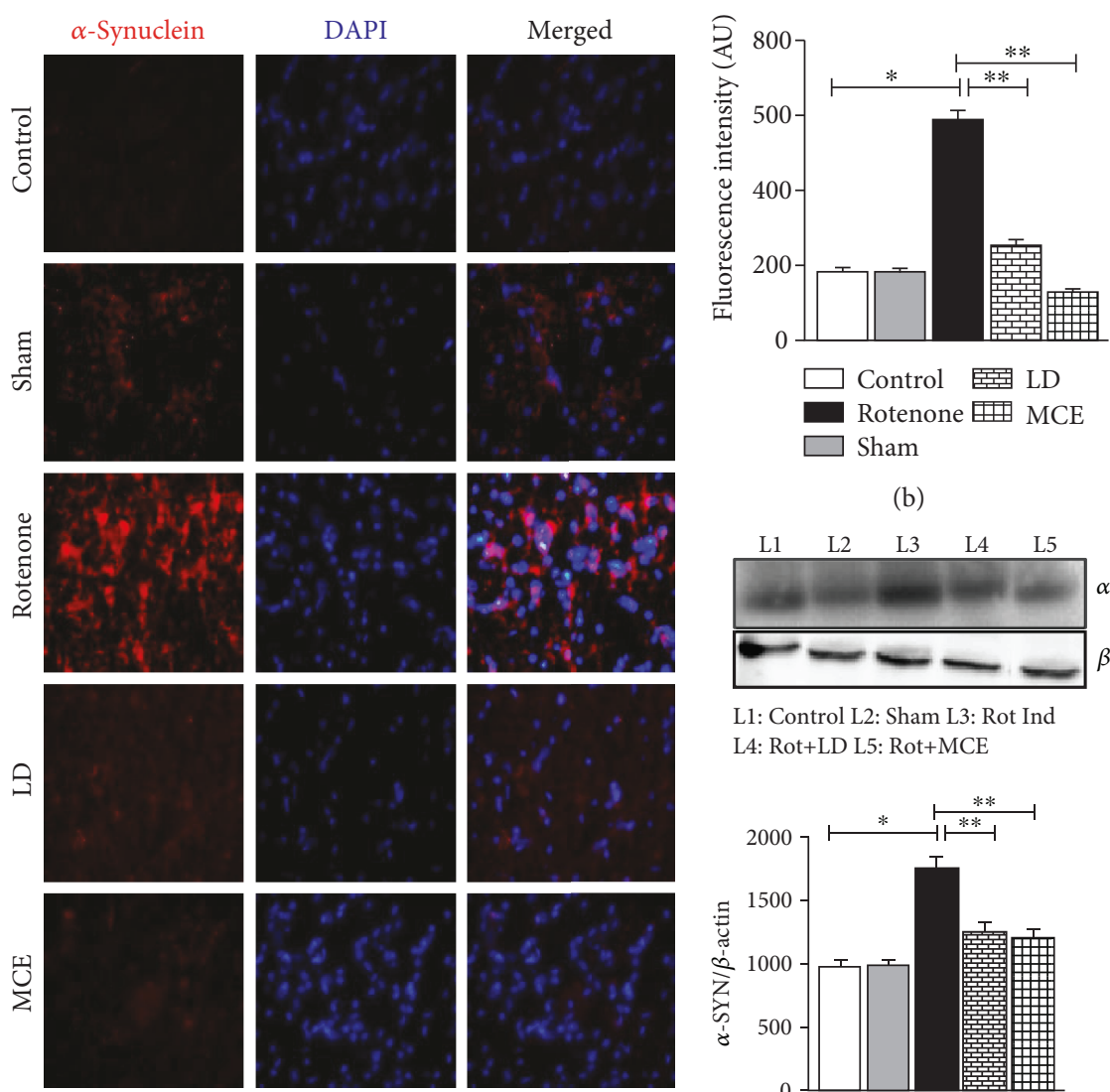

(a)

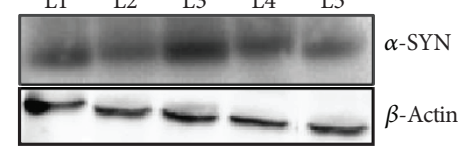

L1: Control L2: Sham L3: Rot Ind L4: Rot+LD L5: Rot+MCE

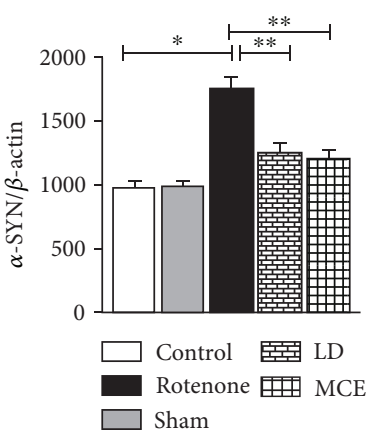

(c)

FIGURE 2: Rotenone-induced aggregation of $\alpha$-synuclein ( $\alpha$-Syn) measured by immunofluorescence analysis. (a) The cells were visualized using fluorescence microscopy and images captured using 20x magnification. The control and sham groups show very low level of expression; however, the expression is punctate and high in the rotenone-induced group; the expression is meager in levodopa; on the contrary, expression in the MCE groups is comparable with that in the control group. (b) Relative fluorescence intensity was calculated. (c) Immunoblot analysis of $\alpha$-Syn and representative densitometry quantification. Statistical significance $(p<0.05)$ was calculated by Student-Newman-Keuls and least significant difference post hoc test, where ${ }^{*}$ represents control vs. other groups, ${ }^{* *}$ represents rotenone vs. LD, MCE.

and its interacting proteins to test the hypothesis that the MCE-mediated augmentation of antioxidative system occurs through the activation of Nrf2/ARE signaling.

Immunoblotting analysis revealed that rotenone infusion significantly decreased Nrf2 protein levels. In compounding fashion, Keap1 and Cullin3, scaffold and adaptor proteins responsible for cytosolic sequestration and proteasomal degradation of $\mathrm{Nrf} 2$, respectively, were significantly increased in rotenone-infused rats. Interestingly, MCE treatment significantly rescued the levels of $\mathrm{Nrf} 2$ and reversed the rotenone-induced increase in Keap1 and Cullin3 (Figure 3(a)). Recent reports indicate that nuclear translocation of Nrf2 is not only mediated by phosphorylation by PKC- $\delta$ but also by intact DJ-1, which binds and stabilizes the Nrf2 and favours its translocation to the nucleus [16]. Next, we extended our immunoblotting analyses to DJ-1; similar to that of Nrf2, the DJ-1 is also downregulated in rotenone-infused rats and this was reversed upon MCE administration (Figure 3(a)). To delineate the difference in total protein expression, we performed the immunoblotting of Nrf2 and Keap1 in nuclear and cytosolic fractions (Figure 3(b)). Our results indeed confirmed that rotenone infusion significantly repressed the translocation of $\mathrm{Nrf} 2$ from the cytosol to the nucleus as evident from decreased levels of nuclear Nrf2 in rotenone versus control rats. Hence, rotenone infusion not only decreases total Nrf2 protein levels but also impairs its nuclear translocation by downregulating DJ-1 and augmenting cytosolic Keap1 levels. As such, supplementation with MCE significantly augmented the nuclear translocation of $\mathrm{Nrf} 2$ as evident from increased levels of nuclear Nrf2 (83\%) by augmenting the DJ-1 and decreasing the nuclear Keap1 levels when compared with rotenone-infused rats.

3.6. MCE Augments Nrf2/ARE Downstream Genes. Nrf2, a member of Cap'n'Collar family of basic region-leucine zipper (bZIP) transcription factors, plays an important role in ARE-mediated gene expression through the transcriptional 
TABle 1: (a) Impact of MCE on antioxidant defense system. Values are expressed as mean \pm SEM for six animals in each group. SOD: units/mg protein. One unit is equal to the amount of enzyme that inhibits the pyrogallol autoxidation by $50 \%$; $\mathrm{CAT}: \mu$ moles of $\mathrm{H}_{2} \mathrm{O}_{2}$ consumed/min/mg protein; GPx: $\mu \mathrm{g}$ of GSH consumed/min/mg protein; GR: nmoles of NADPH oxidized/min/mg protein; GST: $\mu \mathrm{moles}$ of CDNB conjugate formed/minute/mg protein; GSH: nmoles/mg protein. (b) Impact of MCE on the activities of Nrf2/ARE downstream enzymes. Values are expressed as mean \pm SEM for six animals in each group. $\gamma$ GCLC: millimoles of NADH oxidized/min/mg protein; NQO1: nmoles DCPIP utilized/min/mg protein; HO-1: nmoles of bilirubin/h/mg protein. Statistical significance $(p<0.05)$ was calculated by Student-Newman-Keuls and least significant difference post hoc test, where ${ }^{*}$ represents control vs. other groups, ${ }^{* *}$ represents rotenone vs. LD, MCE.

(a) Impact of MCE on antioxidant defense system

\begin{tabular}{lccccc}
\hline Parameter & Control & Sham control & Rotenone induced & LD & MCE \\
\hline SOD & $0.58 \pm 0.018$ & $0.52 \pm 0.017$ & $0.33 \pm 0.015^{*}$ & $0.41 \pm 0.016^{* *}$ & $0.45 \pm 0.014^{* *}$ \\
CAD & $17.43 \pm 0.60$ & $16.36 \pm 0.36$ & $12.13 \pm 0.38^{*}$ & $14.21 \pm 0.32^{* *}$ & $15.22 \pm 0.41^{* *}$ \\
GPx & $8.25 \pm 0.37$ & $8.22 \pm 0.32$ & $5.66 \pm 0.23^{*}$ & $6.59 \pm 0.31^{* *}$ & $6.64 \pm 0.20^{* *}$ \\
GR & $5.16 \pm 0.25$ & $5.12 \pm 0.22$ & $3.90 \pm 0.15^{*}$ & $4.47 \pm 0.19^{* *}$ & $4.48 \pm 0.15^{* *}$ \\
GST & $9.08 \pm 0.394$ & $9.01 \pm 0.468$ & $6.38 \pm 0.271^{*}$ & $7.75 \pm 0.298^{* *}$ & $7.86 \pm 0.241^{* *}$ \\
GSH & $1.93 \pm 0.055$ & $1.76 \pm 0.048$ & $1.28 \pm 0.058^{*}$ & $1.61 \pm 0.059^{* *}$ & $1.65 \pm 0.062^{* *}$ \\
\hline
\end{tabular}

(b) Impact of MCE on the activities of Nrf2/ARE downstream enzymes

\begin{tabular}{lccccc}
\hline Parameter & Control & Sham control & Rotenone induced & LD & MCE \\
\hline$\gamma$ GCL & $2.54 \pm 0.084$ & $2.46 \pm 0.083$ & $1.35 \pm 0.051^{*}$ & $1.90 \pm 0.071^{* *}$ & $2.01 \pm 0.068^{* *}$ \\
NQO1 & $5.88 \pm 0.193$ & $5.85 \pm 0.193$ & $3.15 \pm 0.111^{*}$ & $4.57 \pm 0.151^{* *}$ & $4.82 \pm 0.153^{* *}$ \\
HO-1 & $0.81 \pm 0.026$ & $0.80 \pm 0.026$ & $0.48 \pm 0.016^{*}$ & $0.63 \pm 0.021^{* *}$ & $0.65 \pm 0.018^{* *}$ \\
\hline
\end{tabular}

activation of antioxidant genes such as heme oxygenase-1 (Ho-1), gamma-glutamyl cysteine ligase $(\gamma \mathrm{Gclc})$, and $\mathrm{NAD}(\mathrm{P}) \mathrm{H}$ :quinone oxidoreductase 1 (Nqo1). As we observed a significant decline in Nrf2 protein levels, we further assayed transcript and protein levels for Nrf2 gene targets. Consistent with our observation of oxidative insult and Nrf2 pathway antagonism, rotenone treatment was associated with a prominent decline in the mRNA (Figure 4(a)) and protein (Figure 4(b)) levels of several ARE targets with a maximum decrease being observed in Ho-1 (45\%). However, supplementation with MCE significantly enhanced the levels of these proteins both at the transcriptional and translational levels by an average of $40 \%$. The improved protein levels are reflected in the activities of these enzymes (Table $1(\mathrm{~b})$ ).

After characterizing the neuroprotective effect of MCE in an in vivo model of $\mathrm{PD}$ and confirming its ability to augment Nrf2 signaling and reverse oxidative insult, we further examined the neuroprotective efficacy of scopoletin, a major and active principle of MCE using SH-SY5Y cells. To choose an optimal concentration of scopoletin for this study, we pretreated SH-SY5Y neuroblastoma cells with different doses of scopoletin ranging from 1 to $100 \mu \mathrm{M}$ at different time intervals (from 0 hours to 6 hours). Until $50 \mu \mathrm{M}$ scopoletin, there was no toxicity observed in SH-SY5Y cells. Maximum viability was achieved at the concentration of $30 \mu \mathrm{M}$ scopoletin (Supplementary Figure 2). Hence, further studies were carried out using $30 \mu \mathrm{M}$ scopoletin.

3.7. Scopoletin Prevented Rotenone-Induced Cell Death. SHSY5Y cells undergoing various stages of apoptosis (early, midstage, and late stage) were analyzed by flow cytometry using annexin $\mathrm{V}$ and propidium iodide (PI) dual staining. Treatment of SH-SY5Y cells with rotenone $(500 \mathrm{nM}$ for $24 \mathrm{~h}$ ) resulted in $40 \%$ cell death, which was attenuated by pretreating the cells with $30 \mu \mathrm{M}$ scopoletin for $3 \mathrm{~h}$ (Figures 5(a) and 5(b)). These data indicate that scopoletin protects SH-SY5Y from rotenone-induced cell death.

3.8. Scopoletin-Mediated Neuroprotective Effects Were Associated with the Translocation of Nuclear p40Nrf2 and Upregulation of DJ-1. The activation of the Nrf2/ARE pathway is known to confer resistance to oxidative stress-induced cell death [17]. As scopoletin prevented rotenone-induced cell death, we further assessed Nrf2 pathway constituents. Along these lines, both the unphosphorylated (total/cytosolic form) and the serine 40-phosphorylated Nrf2 (pNrf2S40) levels were measured by immunoblotting. Consistent with animal studies demonstrating that MCE improves nuclear levels of Nrf2, scopoletin also increased the nuclear translocation of Nrf2 as evident from the increased nuclear levels of pNrf2 (S40) (Figure 5(d)). This increase in the nuclear levels of Nrf2 may be attributed to the phosphorylation of Nrf2 by PKC- $\delta$ which is also augmented upon pretreating with scopoletin (Figure 5(c)). Concomitant with the animal studies, both the total and nuclear levels of Keap1 were significantly elevated in rotenone-treated SH-SY5Y cells when compared with untreated control cells. Furthermore, rotenone treatment also increased the levels of E3 ubiquitin ligase Cullin3 (Figure $5(\mathrm{c})$ ) by $46 \%$.

In order to determine whether DJ-1 plays an important role in scopoletin-mediated Nrf2 translocation, we further 

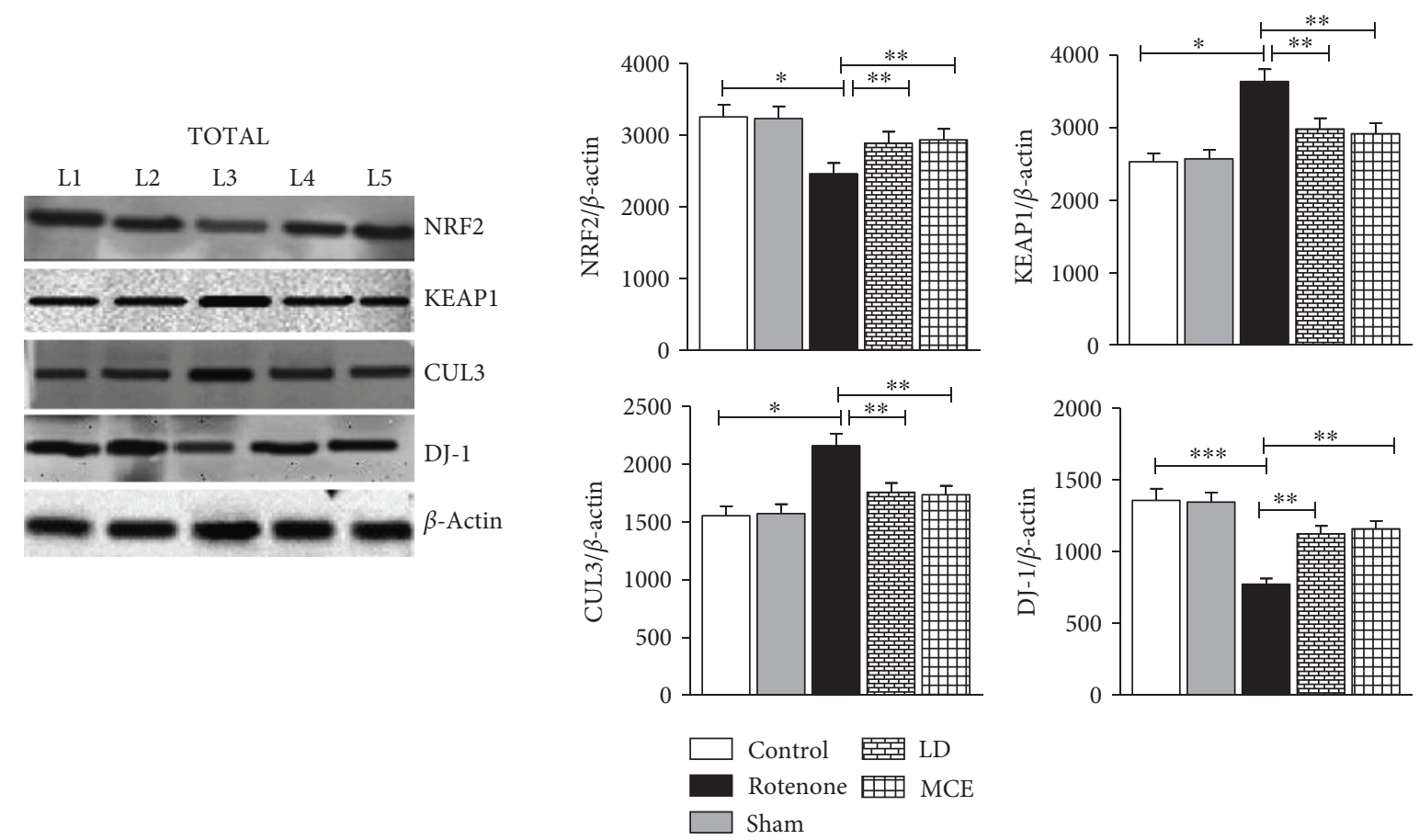

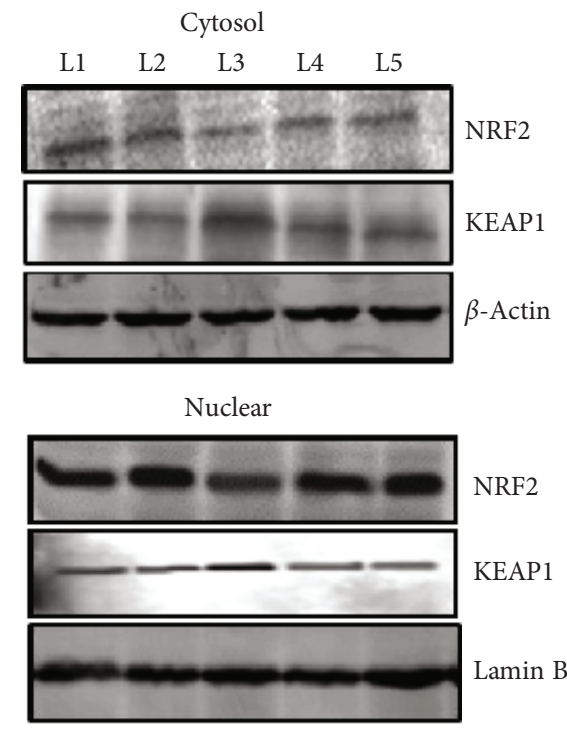

L1: Control L2: Sham L3: Rot Ind L4: Rot+LD L5: Rot+MCE

(a)
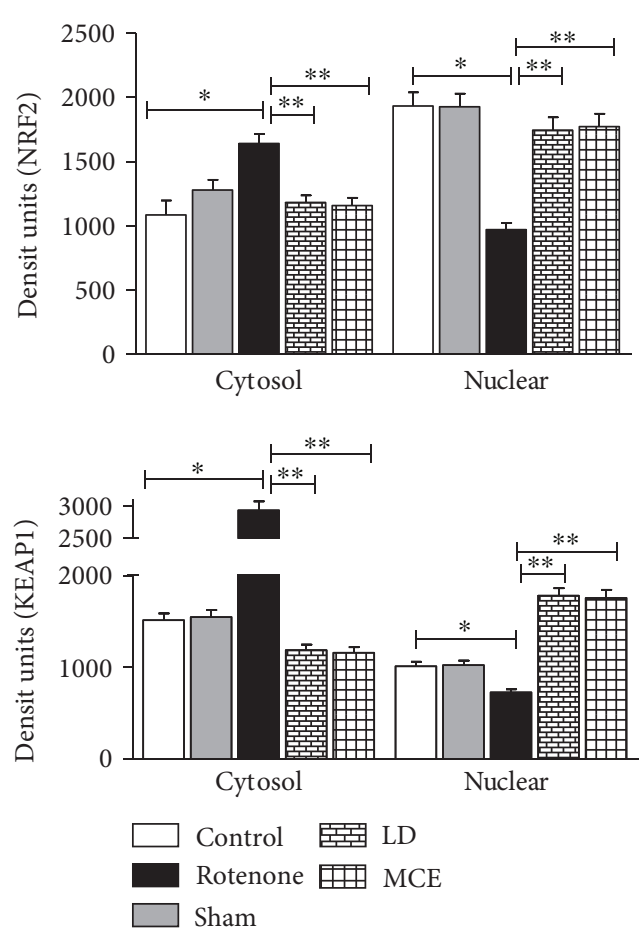

(b)

FIGURE 3: Immunoblot analysis of Nrf2 and its negative regulators Keap1 and Cullin3 in the striatum of rotenone-induced PD rats. (a) The total protein levels of Nrf2, Keap1, Cullin3, and DJ-1. (b) The protein levels of Nrf2 and Keap1 in cytosolic and nuclear compartment of striatal neuronal cells. Statistical significance $(p<0.05)$ was calculated by Student-Newman-Keuls and least significant difference post hoc test, where ${ }^{*}$ represents control vs. other groups, ${ }^{* *}$ represents rotenone vs. LD, MCE.

analyzed the levels of DJ-1 in rotenone-induced SH-SY5Y cells pretreated with or without scopoletin. Rotenonetreated cells exhibited a relative decrease in the protein levels of DJ-1 (Figure 5(c)) which may be attributed to the impaired Nrf2 nuclear translocation in these cells. Conversely, scopoletin-pretreated cells showed an augmented 
A
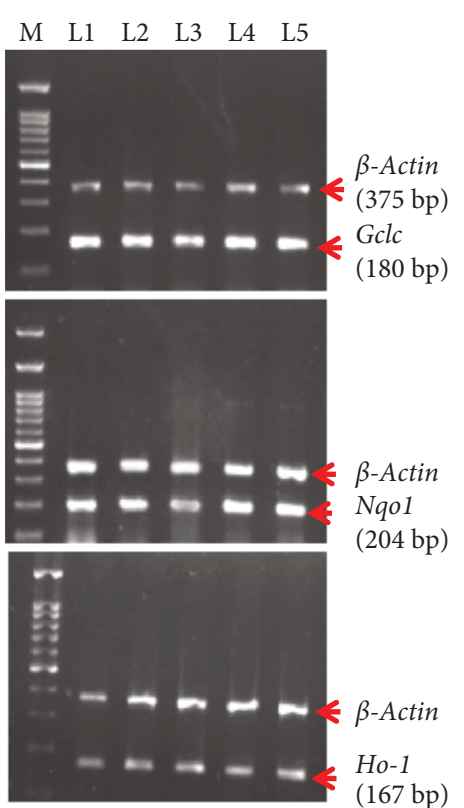

$\beta$-Actin

Nqo1

(204 bp)
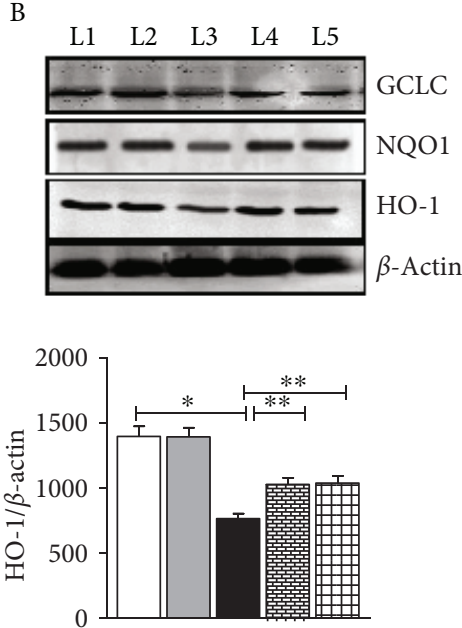

M- DNA Ladder L1: Control L2: Sham L3: Rotenone L4: Rot+LD L5: Rot+MCE
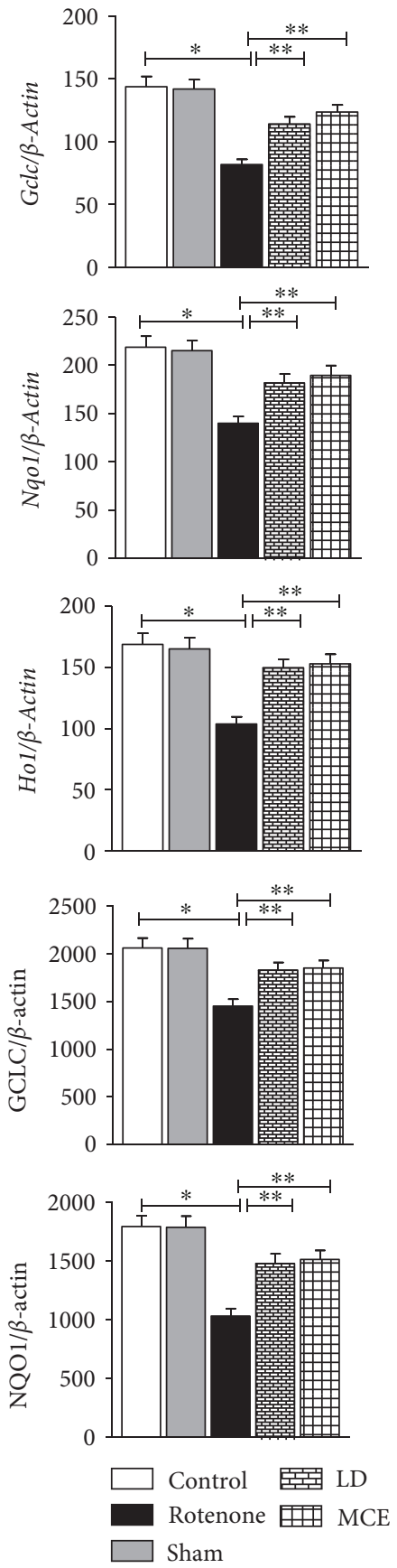

FIGURE 4: Increased Nrf2 regulated antioxidant genes in response to MCE supplementation in rotenone-induced PD rats. (a) The mRNA levels of Nrf2 downstream genes $\gamma$ GCLC, NQO1, and HO-1. (b) The protein levels of $\gamma$ GCLC, NQO1, and HO-1. Statistical significance $(p<0.05)$ was calculated by Student-Newman-Keuls and least significant difference post hoc test, where ${ }^{*}$ represents control vs. other groups, ${ }^{* *}$ represents rotenone vs. LD, MCE.

expression of DJ-1 (46\%), suggesting that DJ-1 may be vital for scopoletin-mediated neuroprotective effects. From these observations, it is clear that scopoletin augments the Nrf2/ARE pathway by increasing levels of DJ-1 and concomitantly prevents the cytosolic degradation of $\mathrm{Nrf} 2$ by reducing the levels of its negative regulators Keap1 and Cullin3.

\section{Discussion}

Increasing interest has been focused on identifying dietary supplements and phytoconstituents that can inhibit ROSmediated protein aggregation and neuronal cell death and thereby reverse the multifaceted pathophysiological events underlying PD. Here, we investigated whether Morinda 


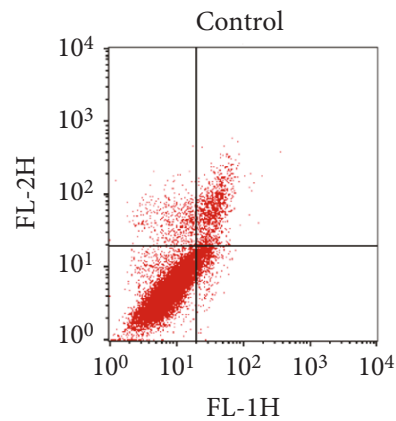

FL-1H

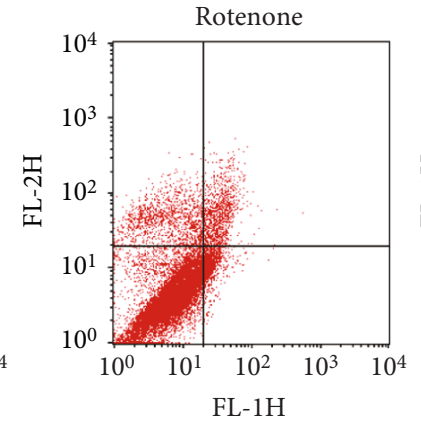

(a)

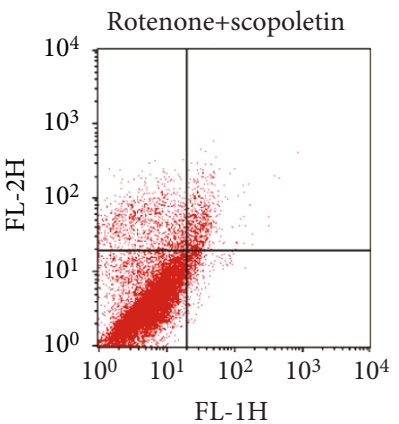

FL-1H

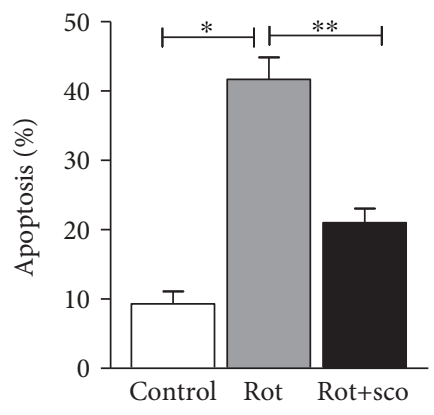

(b)
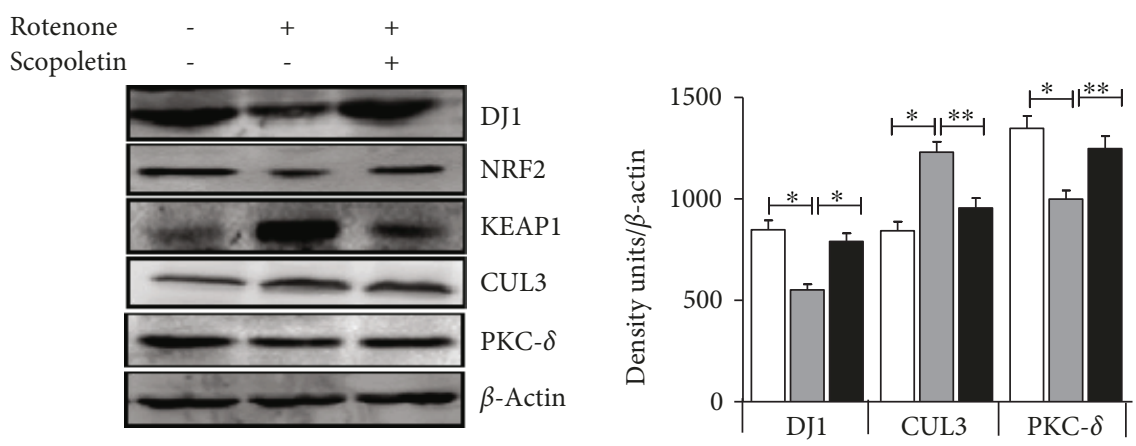

(c)
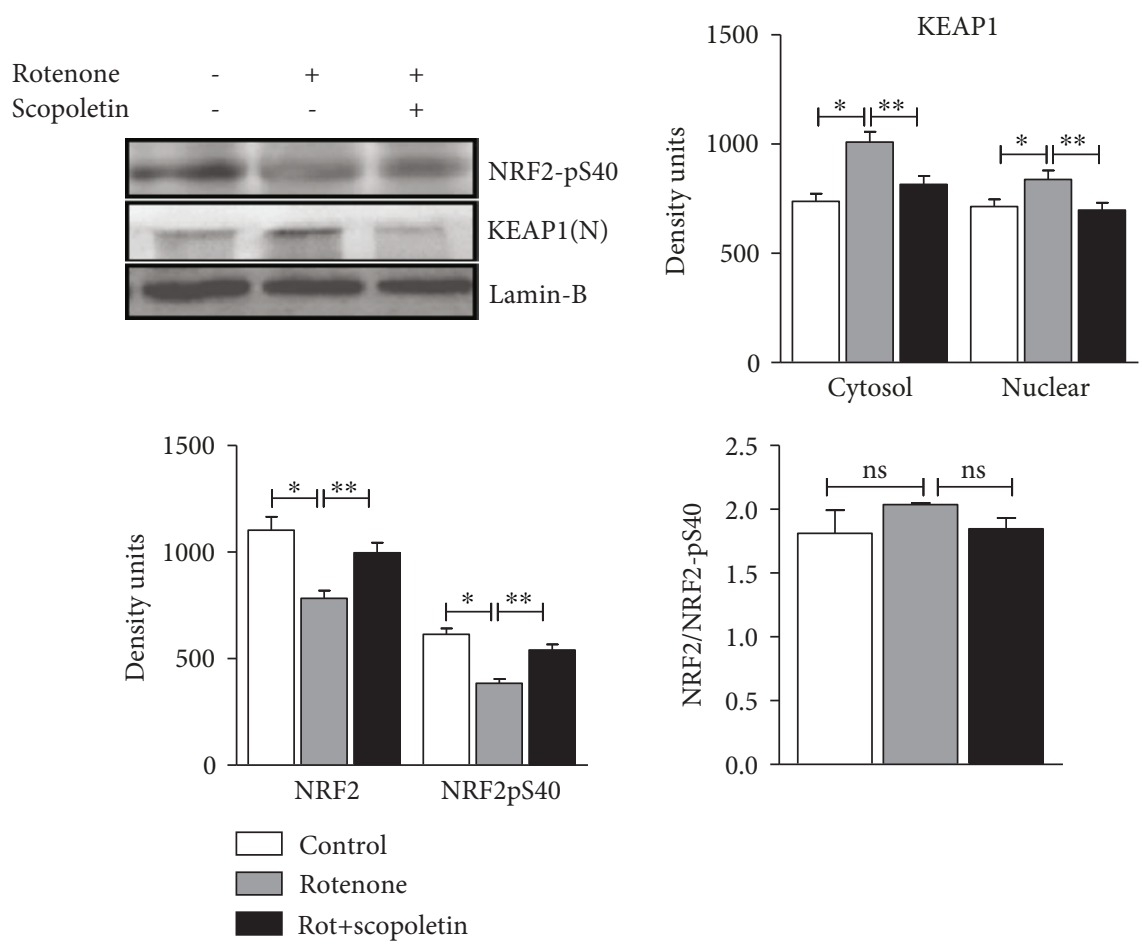

(d)

FIGURE 5: (a) Rotenone-induced apoptosis is counteracted by scopoletin in SH-SY5Y cells. At the end of the experiment, cells were stained with annexin V/FITC and read immediately by flow cytometry to measure the extent of apoptosis; $x$-axis FL-1H denotes the FITC and $y$ -axis FL-2H denotes the PI. (b) Quantification of percentage dead cells. (c) Immunoblot analysis of several proteins involved in Nrf2/ARE pathway is performed in SH-SY5Y cells treated with/without rotenone and scopoletin. (d) Immunoblot analysis of phospho (S40) Nrf2 and nuclear Keap1 in SH-SY5Y cells. Statistical significance $(p<0.05)$ was calculated by Student-Newman-Keuls and least significant difference post hoc test, where ${ }^{*}$ represents control vs. other groups, ${ }^{* *}$ represents rotenone vs. scopoletin. 
citrifolia fruit extract attenuates rotenone-induced oxidative stress by activating the Nrf2-dependent antioxidant response and tested whether this mechanism may prevent the loss of dopaminergic neurons. After confirming its neuroprotective effect in vivo, we elucidated the mechanism of action for scopoletin, a major compound present in the $\mathrm{MCE}$, in vitro using SH-SY5Y cells and identified that the therapeutic effect of scopoletin is facilitated through the activation of the DJ-1/Nrf2/ARE signaling cascade.

While the pathogenic mechanism of PD is poorly known, it is believed that oxidative stress involving the imbalance of nitric oxide (NO) signaling is a major player in the prognosis of PD [14]. A significant increase in the levels of NO in the striatum of rotenone-infused Parkinsonian rats is in line with previous reports [18]. However, supplementation of MCE reduced the levels of NO in the striatum of PD-induced rats. Recent reports documented that the ethyl acetate extract of noni fruit is shown to reduce oxidative and nitrosative stress in the brain [19]. As NO homeostasis is significantly impaired in response to rotenone infusion, our further analysis revealed an increase in the levels of iNOS in the striatal tissues in these rats. Thus, the increased iNOS might have been responsible for increased nitric oxide levels. The administration of MCE safeguarded the striatum from the deleterious effect of nitric oxide by attenuating the iNOS expression induced by rotenone (Figure 1).

As protein aggregation is a common event underlying neurodegenerative diseases including $\mathrm{PD}$, wherein $\alpha$-synuclein $(\alpha$-Syn) comprises the bulk of Lewy bodies [20, 21], further investigations were directed to assess the rate of $\alpha$ Syn aggregation. $\alpha$-Syn expression and its aggregation were increased in the striatum of rotenone-infused rats when compared with the control rats (Figure 2). This increased aggregation of $\alpha$-Syn might also be due to NO-mediated oxidative stress in PD rats (Table 1(a)), as it has been previously shown that fibrillary $\alpha$-Syn aggregates with perinuclear localization were formed in cells exposed to NO [22]. Interestingly, treatment with MCE reduced the $\alpha$-Syn aggregation which might be due to the reduction in the levels of $\mathrm{NO}$ and the consequent tyrosine nitration in MCE-treated rats.

Intracellular defense is maintained through a variety of antioxidant enzymes and low molecular weight antioxidants such as glutathione to combat the deleterious effects of ROS overproduction and oxidative damage [23]. Elevated ROS production, in the absence of increased antioxidant defenses, will exacerbate oxidative damage and oxidative stress [24]. In the current study, an overall decline in the activities of antioxidant enzymes such as superoxide dismutase (SOD), catalase (CAT), glutathione peroxidase (GPx), glutathione reductase (GR), and glutathione-s-transferase (GST) was noticed along with significantly decreased glutathione (GSH) content in the striatum of intranigrally rotenoneinfused rats when compared with control rats. Notably, MCE supplementation restored the overall antioxidant status, rescuing glutathione levels in the striatum of rotenone-infused PD rats. These results support recent work demonstrating that the ethyl acetate extract of Morinda citrifolia fruit boosts SOD, GPx, and GR enzymatic activity in $\beta$-amyloid-induced cognitive dysfunction in mice [25] and in the skeletal muscle of rotenone-infused hemiParkinsonian rats [10].

In the present investigation, the total protein level of Nrf2 was significantly reduced in rotenone-induced rats while Keap1 and Cullin3, negative regulators of Nrf2, were markedly elevated. High levels of oxidative stress may reduce the activity of Nrf2, although the molecular mechanism for this defect is uncertain [26]. Interestingly, the administration of MCE reversed the rotenone-mediated increase in Keap1 and Cullin3, thereby preventing the degradation of Nrf2 in vivo. Keap1 is capable of restraining Nrf2 activity not only via its capacity to target Nrf2 to a cytoplasmic Cullin3-based E3 ligase [27] but also by transiently entering into the nucleus and targeting Nrf2 for ubiquitylation in this compartment under stressed conditions [28]. This instigated us to analyze the nuclear levels of Nrf2 and Keap1 which gives us an unblemished picture of the nuclear translocation and activated form of Nrf2.

The nuclear level of Nrf2 was reduced in the striatum of rotenone-infused Parkinsonian rats when compared with the control rats and the opposite trend was observed for Keap1. From these observations, it may be inferred that not only is a lower concentration of Nrf2 protein present in the nucleus but that it also may be bound by Keap1, thereby preventing Nrf2 from binding to AREs. Indeed, this notion is consistent with our observation of a lower antioxidant status in these rats. However, the redox milieu inside the striatal nucleus of PD rats treated with MCE is different, where MCE with its rich phytoconstituents favours the translocation of Nrf2 into the nucleus and detains Keap1 in the cytosol, which is further reflected in the results obtained for antioxidant status in these rats. From these observations, it is clear that MCE increases the nuclear translocation of Nrf2.

Since we observed a significant decline in the transcriptional activity of Nrf2, we further assessed its downstream effectors ( $\gamma$ Gclc, Nqo1 and Ho-1) by analyzing the levels of mRNA and protein. Overall, both the mRNA and protein levels of $\gamma \mathrm{Gclc}$, Nqo1 and Ho-1 were significantly decreased in the striatum of rats stereotaxically infused with rotenone when compared with the control rats. The consequence of impaired transcription and translation of these proteins was also reflected in the diminished activities of these enzymes on rotenone infusion. However, upon treating with MCE, the activities and mRNA and protein levels of these proteins were significantly increased, likely a downstream effect of enhanced Nrf2 stability and activation.

Overall, from the in vivo studies, it is clear that MCE, with its antioxidant property, scavenges the free radicals, and it also reduces the expression of iNOS and prevents rotenoneinduced aggregation of $\alpha$-synuclein. MCE also augments the total levels of Nrf2 and subsequently translocates Nrf2 to the nucleus by preventing its degradation mediated by Keap1/Cullin3 complex which in turn leads to transcription and enhanced activities of its downstream effectors $\gamma \mathrm{Gclc}$, Ho-1 and Nqo1. This neuroprotective effect of MCE might be attributed to the presence of identified phytoconstituents 
quercetin, rutin, and scopoletin and also other unidentified constituents [10]. However, as scopoletin is the biomarker for Morinda citrifolia [29] while other compounds such as rutin and quercetin are commonly present in most of the plants $[30,31]$, further emphasis was given to scopoletin, and its mechanism of action in boosting DJ-1/Nrf2/ARE pathway was studied in in vitro cell culture using SH-SY5Y dopaminergic cells, in order to delineate the ability of the phytochemical derived from Morinda citrifolia on dopaminergic neuronal cell survival.

We observed a significant reduction in cell viability on rotenone-exposed SH-SY5Y cells (data not shown), which was attenuated by pretreatment with scopoletin. Hence, we further analyzed the status of cellular apoptosis using annexin V/propidium iodide staining. Rotenone exposure of SH-SY5Y cells shows a significant increase in the proportion of early apoptotic cells compared with the controls. Our observation is in coherence with the previous reports by Jang et al. [32], who have stated that rotenone at a concentration of $200 \mathrm{nM}$ induces apoptosis in SH-SY5Y cells by generating ROS. However, this shift is reversed when the cells are pretreated with scopoletin, and the possible explanation for this effect would be scopoletin with its antioxidant potential [33] should have ameliorated rotenone-induced apoptosis by quenching free radicals. To the best of our knowledge, this is the first study to show that scopoletin prevents apoptosis in an in vitro rotenone exposure unless otherwise like literature which poses it as a potent proapoptotic agent in various cancer cell lines [34-36].

In light of our observations on MCE-mediated Nrf2 activation, we examined the capacity of scopoletin to influence the Nrf2 signaling by aiding the nuclear translocation of Nrf2. We analyzed the levels of phospho-Nrf2 and PKC- $\delta$ that phosphorylate serine 40 of Nrf2, thereby aiding in its nuclear translocation, in SH-SY5Y cells exposed to rotenone. Pretreatment with scopoletin augmented the nuclear levels of phospho-Nrf2 which may be associated with our observation of increased PKC- $\delta$ expression. Indeed, Nam and Kim [37] have shown that scopoletin influences the expression of reprogramming genes and exerts antiaging effects by regulating the transcription factor Nrf2 (Figure 5). Previous reports have shown that DJ-1 stabilizes Nrf2 and promotes its nuclear translocation [38]. Intriguingly, mutations in DJ-1 are associated with the risk of developing PD [39]. Hence, we further evaluated the effect of scopoletin on DJ-1 levels in SH-SY5Y cells. We found that rotenone exposure of cells resulted in diminished levels of DJ-1. Angeline et al. [40] have reported that chronic exposure to rotenone reduced the cytoprotective proteins Parkin, Hsp70, and DJ-1. However, on pretreating with scopoletin, DJ-1 protein levels were augmented, subsequently conferring protection against rotenone-induced oxidative stress, as overexpression of DJ-1 rescued MN9D cells exposed to rotenone, indicating that DJ-1 protected nigral DA neurons from rotenone-induced cell death [41].

\section{Conclusions}

This study has provided evidence that MCE prevented $\alpha$ synuclein aggregation through augmenting Nrf2 antioxidant signaling, leading to the suppression of oxidative stress. Notably, scopoletin, the active component from MCE, seems to be responsible for stabilizing Nrf2/ARE pathway by augmenting the phosphorylation of $\mathrm{Nrf} 2$ and its nuclear translocation, in a DJ-1-dependent manner. Thus, we propose that DJ-1 might be a potential target for scopoletin-based therapeutic strategy against neurodegenerative diseases.

\author{
Abbreviations \\ DMSO: Dimethyl sulfoxide \\ F-12K: Kaighn's modification of Ham's F-12 medium \\ FBS: $\quad$ Fetal bovine serum \\ GSH: $\quad$ Reduced glutathione \\ HO-1: Heme oxygenase-1 \\ Keap1: Kelch-like erythroid cell-derived protein with \\ CNC (Cap "n" Collar) homology (ECH) protein1 \\ LD: Levodopa \\ LPO: Lipid peroxidation \\ MCE: $\quad$ Ethyl acetate extract of Morinda citrifolia fruit \\ NO: $\quad$ Nitric oxide \\ NQO1: $\quad \mathrm{NAD}(\mathrm{P}) \mathrm{H}$ :quinone oxidoreductase 1 \\ Nrf2: $\quad$ Nuclear factor erythroid 2-related factor 2 \\ PC: $\quad$ Protein carbonyl \\ PD: $\quad$ Parkinson's disease \\ PKC- $\delta$ : $\quad$ Protein kinase $C$ delta \\ ROS: $\quad$ Reactive oxygen species \\ Rot Ind: Rotenone induced \\ SH-SY5Y: Neuroblast-like subclone of SK-N-SH \\ SNPc: $\quad$ Substantia nigra pars compacta \\ VTA: Ventral tegmental area \\ $\gamma$ GCLC: Catalytic subunit of gamma glutamate cysteine \\ ligase.
}

\section{Data Availability}

The data supporting the findings of this study are available within the article [and/or] its supplementary materials.

\section{Ethical Approval}

All experiments were performed in accordance with the guidelines approved by the Institutional Animal Ethical Committee (IAEC No. 01/09/12, 01/09/12 Extension).

\section{Conflicts of Interest}

The authors declare that they have no potential conflict of interest including any financial and personal relationships with other people or organizations.

\section{Authors' Contributions}

Kishore Kumar S. Narasimhan, the first author, designed and executed the experimental work; collected, analyzed, and interpreted the data; and drafted the manuscript. Deepthy Jayakumar and Saraswathi Uthamaraman assisted with key experiments and were involved in data collection. Kishore Kumar S. Narasimhan, Prema Velusamy, and Ashokkumar Srinivasan were responsible for the in vitro and flow 
cytometry experiments. Thangarajeswari Mohan, Divya Bhavani Ravi, and Yogesh Kanna Sathyamoorthy were responsible for immunofluorescence analysis and quantification and language editing. Namakkal Soorappan Rajasekaran revised the article and provided important directions for interpreting the results and discussion. Kalaiselvi Periandavan conceived the idea, provided directions for designing the experiments, and supervised the entire work.

\section{Acknowledgments}

The financial assistance to Dr. Kishore Kumar S Narasimhan, Department of Medical Biochemistry, from the Indian Council of Medical Research (ICMR) in the form of ICMR Senior Research Fellow (SRF), New Delhi, Government of India, is gratefully acknowledged. Authors greatly appreciate Dr. Gobinath Shanmugam for assisting with artwork (figures) and Mr. Justin Quiles for his editorial assistance.

\section{Supplementary Materials}

The supplementary materials (SM) provided along with this manuscript details about the methods we used to determine the levels of oxidative stress markers, activities of antioxidant defense systems/downstream enzymes of Nrf2/ARE signaling pathway, immunofluorescence of $\alpha$ synuclein, and sequence of the primers used in the PCR protocol. In addition, the SM also provides a data for behavioural analysis (Supplementary Figure 1) and dose determination of scopoletin using MTT assay (Supplementary Figure 2). (Supplementary Materials)

\section{References}

[1] B. J. Ryan, S. Hoek, E. A. Fon, and R. Wade-Martins, "Mitochondrial dysfunction and mitophagy in Parkinson's: from familial to sporadic disease," Trends in Biochemical Sciences, vol. 40, no. 4, pp. 200-210, 2015.

[2] X. Chen, C. Guo, and J. Kong, "Oxidative stress in Neurodegenerative diseases," Neural Regeneration Research, vol. 7, no. 5, pp. 376-385, 2012.

[3] T. Tamura, M. Yoshida, Y. Hashizume, and G. Sobue, "Lewy body-related $\alpha$-synucleinopathy in the spinal cord of cases with incidental Lewy body disease," Neuropathology, vol. 32, no. 1, pp. 13-22, 2012.

[4] S. Shendelman, A. Jonason, C. Martinat, T. Leete, and A. Abeliovich, "DJ-1 is a redox-dependent molecular chaperone that inhibits $\alpha$-synuclein aggregate formation," PLoS Biology, vol. 2, no. 11, article e362, 2004.

[5] L. Gan and J. A. Johnson, "Oxidative damage and the Nrf2ARE pathway in neurodegenerative diseases," Biochimica et Biophysica Acta (BBA) - Molecular Basis of Disease, vol. 1842, no. 8, pp. 1208-1218, 2014.

[6] F. Jin, Q. Wu, Y. F. Lu, Q. H. Gong, and J. S. Shi, "Neuroprotective effect of resveratrol on 6-OHDA-induced Parkinson's disease in rats," European Journal of Pharmacology, vol. 600, no. 1-3, pp. 78-82, 2008.
[7] J. A. Johnson, D. A. Johnson, A. D. Kraft et al., “The Nrf2-ARE pathway," Annals of the New York Academy of Sciences, vol. 1147, no. 1, pp. 61-69, 2008.

[8] Y. Masaki, Y. Izumi, A. Matsumura, A. Akaike, and T. Kume, "Protective effect of Nrf2-ARE activator isolated from green perilla leaves on dopaminergic neuronal loss in a Parkinson's disease model," European Journal of Pharmacology, vol. 798, pp. 26-34, 2017.

[9] V. Sharma and A. Agarwal, "Physicochemical and antioxidant assays of methanol and hydromethanol extract of ariel parts of Indigofera tinctoria Linn," Indian Journal of Pharmaceutical Sciences, vol. 77, no. 6, pp. 729-734, 2015.

[10] K. K. S. Narasimhan, L. Paul, Y. K. Sathyamoorthy et al., "Amelioration of apoptotic events in the skeletal muscle of intra-nigrally rotenone-infused Parkinsonian rats by Morinda citrifolia - up-regulation of Bcl-2 and blockage of cytochrome c release," Food \& Function, vol. 7, no. 2, pp. 922 937, 2016.

[11] S. N. Kishore Kumar, J. Deepthy, U. Saraswathi et al., "Morinda citrifolia mitigates rotenone-induced striatal neuronal loss in male Sprague-Dawley rats by preventing mitochondrial pathway of intrinsic apoptosis," Redox Report, vol. 22, no. 6, pp. 418-429, 2017.

[12] C. E. Adams, A. F. Hoffman, J. L. Hudson, B. J. Hoffer, and S. J. Boyson, "Chronic treatment with levodopa and/or selegiline does not affect behavioral recovery induced by fetal ventral mesencephalic grafts in unilaterally 6-hydroxydopaminelesioned rats," Experimental Neurology, vol. 130, no. 2, pp. 261-268, 1994.

[13] Y. N. Deng, J. Shi, J. Liu, and Q. M. Qu, "Celastrol protects human neuroblastoma SH-SY5Y cells from rotenoneinduced injury through induction of autophagy," Neurochemistry International, vol. 63, no. 1, pp. 1-9, 2013.

[14] K. J. Barnham, C. L. Masters, and A. I. Bush, "Neurodegenerative diseases and oxidative stress," Nature Reviews Drug Discovery, vol. 3, no. 3, pp. 205-214, 2004.

[15] A. Jazwa, A. I. Rojo, N. G. Innamorato, M. Hesse, J. FernándezRuiz, and A. Cuadrado, "Pharmacological targeting of the transcription factor Nrf2 at the basal ganglia provides disease modifying therapy for experimental Parkinsonism," Antioxidants \& Redox Signaling, vol. 14, no. 12, pp. 2347-2360, 2011.

[16] M. S. Bitar, C. Liu, A. Ziaei, Y. Chen, T. Schmedt, and U. V. Jurkunas, "Decline in DJ-1 and decreased nuclear translocation of Nrf2 in Fuchs endothelial corneal dystrophy," Investigative Ophthalmology \& Visual Science, vol. 53, no. 9, pp. 5806-5813, 2012.

[17] J. W. Yao, J. Liu, X. Z. Kong et al., "Induction of activation of the antioxidant response element and stabilization of Nrf2 by 3-(3-pyridylmethylidene)-2-indolinone (PMID) confers protection against oxidative stress-induced cell death," Toxicology and Applied Pharmacology, vol. 259, no. 2, pp. 227-235, 2012.

[18] Y. He, S. Z. Imam, Z. Dong et al., "Role of nitric oxide in rotenone-induced nigro-striatal injury," Journal of Neurochemistry, vol. 86, no. 6, pp. 1338-1345, 2003.

[19] S. D. Pachauri, P. R. P. Verma, A. K. Dwivedi et al., "Ameliorative effect of Noni fruit extract on streptozotocin-induced memory impairment in mice," Behavioural Pharmacology, vol. 24, no. 4, pp. 307-319, 2013.

[20] L. Stefanis, “ $\alpha$-Synuclein in Parkinson's disease," Cold Spring Harbor Perspectives in Medicine, vol. 2, no. 2, article a009399, 2011. 
[21] A. Oczkowska, W. Kozubski, M. Lianeri, and J. Dorszewska, "Mutations in PRKN and SNCA genes important for the progress of Parkinson's disease," Current Genomics, vol. 14, no. 8, pp. 502-517, 2013.

[22] E. Paxinou, Q. Chen, M. Weisse et al., "Induction of $\alpha$-synuclein aggregation by intracellular nitrative insult," Journal of Neuroscience, vol. 21, no. 20, pp. 8053-8061, 2001.

[23] R. I. López-Cruz, T. Zenteno-Savín, and F. Galván-Magaña, "Superoxide production, oxidative damage and enzymatic antioxidant defenses in shark skeletal muscle," Comparative Biochemistry and Physiology Part A: Molecular \& Integrative Physiology, vol. 156, no. 1, pp. 50-56, 2010.

[24] B. Poljsak, D. Šuput, and I. Milisav, "Achieving the balance between ROS and antioxidants: when to use the synthetic antioxidants," Oxidative Medicine and Cellular Longevity, vol. 2013, Article ID 956792, 11 pages, 2013.

[25] P. Muralidharan, V. R. Kumar, and G. Balamurugan, "Protective effect of Morinda citrifoliafruits on $\beta$-amyloid (25-35) induced cognitive dysfunction in mice: an experimental and biochemical study," Phytotherapy Research, vol. 24, no. 2, pp. 252-258, 2010.

[26] N. Mercado, R. Thimmulappa, C. M. R. Thomas et al., "Decreased histone deacetylase 2 impairs Nrf2 activation by oxidative stress," Biochemical and Biophysical Research Communications, vol. 406, no. 2, pp. 292-298, 2011.

[27] S. B. Cullinan and J. A. Diehl, "PERK-dependent activation of Nrf2 contributes to redox homeostasis and cell survival following endoplasmic reticulum stress," Journal of Biological Chemistry, vol. 279, no. 19, pp. 20108-20117, 2004.

[28] T. Nguyen, P. J. Sherratt, P. Nioi, C. S. Yang, and C. B. Pickett, "Nrf2 controls constitutive and inducible expression of AREdriven genes through a dynamic pathway involving nucleocytoplasmic shuttling by Keap1," Journal of Biological Chemistry, vol. 280, no. 37, pp. 32485-32492, 2005.

[29] S. Mahattanadul, W. Ridtitid, S. Nima, N. Phdoongsombut, P. Ratanasuwon, and S. Kasiwong, "Effects of Morinda citrifolia aqueous fruit extract and its biomarker scopoletin on reflux esophagitis and gastric ulcer in rats," Journal of Ethnopharmacology, vol. 134, no. 2, pp. 243-250, 2011.

[30] A. Wach, K. Pyrzynska, and M. Biesaga, "Quercetin content in some food and herbal samples," Food Chemistry, vol. 100, no. 2, pp. 699-704, 2007.

[31] J. Kalinova and E. Dadakova, "Rutin and total quercetin content in amaranth (Amaranthus spp.)," Plant Foods for Human Nutrition, vol. 64, no. 1, pp. 68-74, 2009.

[32] W. Jang, H. J. Kim, H. Li et al., “1,25-Dyhydroxyvitamin $\mathrm{D}_{3}$ attenuates rotenone-induced neurotoxicity in SH-SY5Y cells through induction of autophagy," Biochemical and Biophysical Research Communications, vol. 451, no. 1, pp. 142-147, 2014.

[33] R. Mogana, K. Teng-Jin, and C. Wiart, "Anti-inflammatory, anticholinesterase, and antioxidant potential of scopoletin isolated from Canarium patentinervium Miq. (Burseraceae Kunth)," Evidence-Based Complementary and Alternative Medicine, vol. 2013, Article ID 734824, 7 pages, 2013.

[34] E. K. Kim, K. B. Kwon, B. C. Shin et al., "Scopoletin induces apoptosis in human promyeloleukemic cells, accompanied by activations of nuclear factor $\kappa \mathrm{B}$ and caspase-3," Life Sciences, vol. 77, no. 7, pp. 824-836, 2005.
[35] P. Zhao, Y. Dou, L. Chen et al., "SC-III3, a novel scopoletin derivative, induces autophagy of human hepatoma HepG2 cells through AMPK/mTOR signaling pathway by acting on mitochondria," Fitoterapia, vol. 104, pp. 31-40, 2015.

[36] Y. M. Tabana, L. E. A. Hassan, M. B. K. Ahamed et al., "Scopoletin, an active principle of tree tobacco (Nicotiana glauca) inhibits human tumor vascularization in xenograft models and modulates ERK1, VEGF-A, and FGF-2 in computer model," Microvascular Research, vol. 107, pp. 17-33, 2016.

[37] H. Nam and M. M. Kim, "Scopoletin has a potential activity for anti-aging via autophagy in human lung fibroblasts," Phytomedicine, vol. 22, no. 3, pp. 362-368, 2015.

[38] C. M. Clements, R. S. McNally, B. J. Conti, T. W. Mak, and J. P. Y. Ting, "DJ-1, a cancer-and Parkinson's disease-associated protein, stabilizes the antioxidant transcriptional master regulator Nrf2," Proceedings of the National Academy of Sciences, vol. 103, no. 41, pp. 15091-15096, 2006.

[39] B. Björkblom, A. Adilbayeva, J. Maple-Grødem et al., "Parkinson disease protein DJ-1 binds metals and protects against metal-induced cytotoxicity," Journal of Biological Chemistry, vol. 288, no. 31, pp. 22809-22820, 2013.

[40] M. S. Angeline, P. Chaterjee, K. Anand, R. K. Ambasta, and P. Kumar, "Rotenone-induced parkinsonism elicits behavioral impairments and differential expression of parkin, heat shock proteins and caspases in the rat," Neuroscience, vol. 220, pp. 291-301, 2012.

[41] H. Gao, W. Yang, Z. Qi et al., "DJ-1 protects dopaminergic neurons against rotenone-induced apoptosis by enhancing ERK-dependent mitophagy," Journal of Molecular Biology, vol. 423, no. 2, pp. 232-248, 2012. 


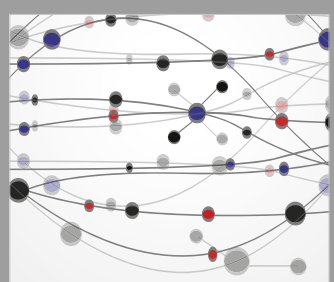

The Scientific World Journal
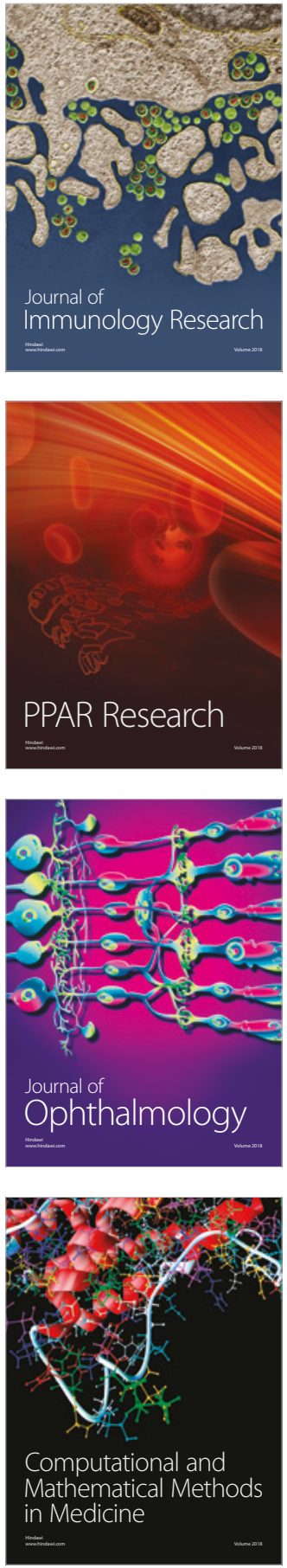

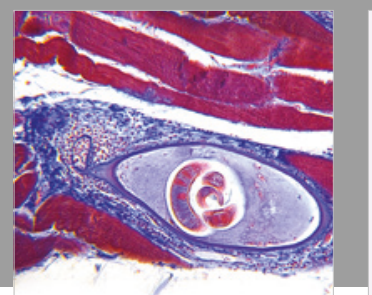

Gastroenterology Research and Practice

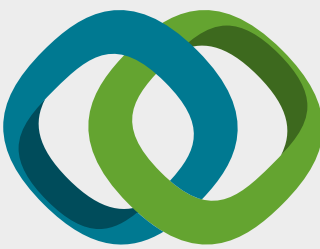

\section{Hindawi}

Submit your manuscripts at

www.hindawi.com
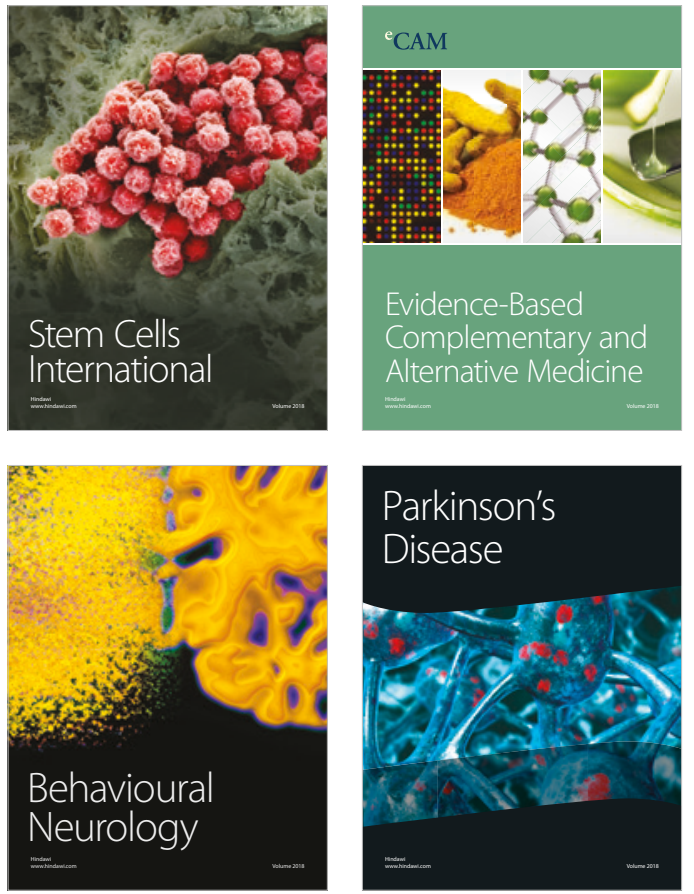

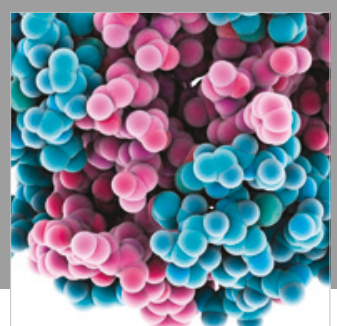

ournal of

Diabetes Research

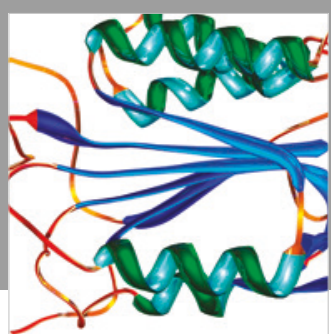

Disease Markers
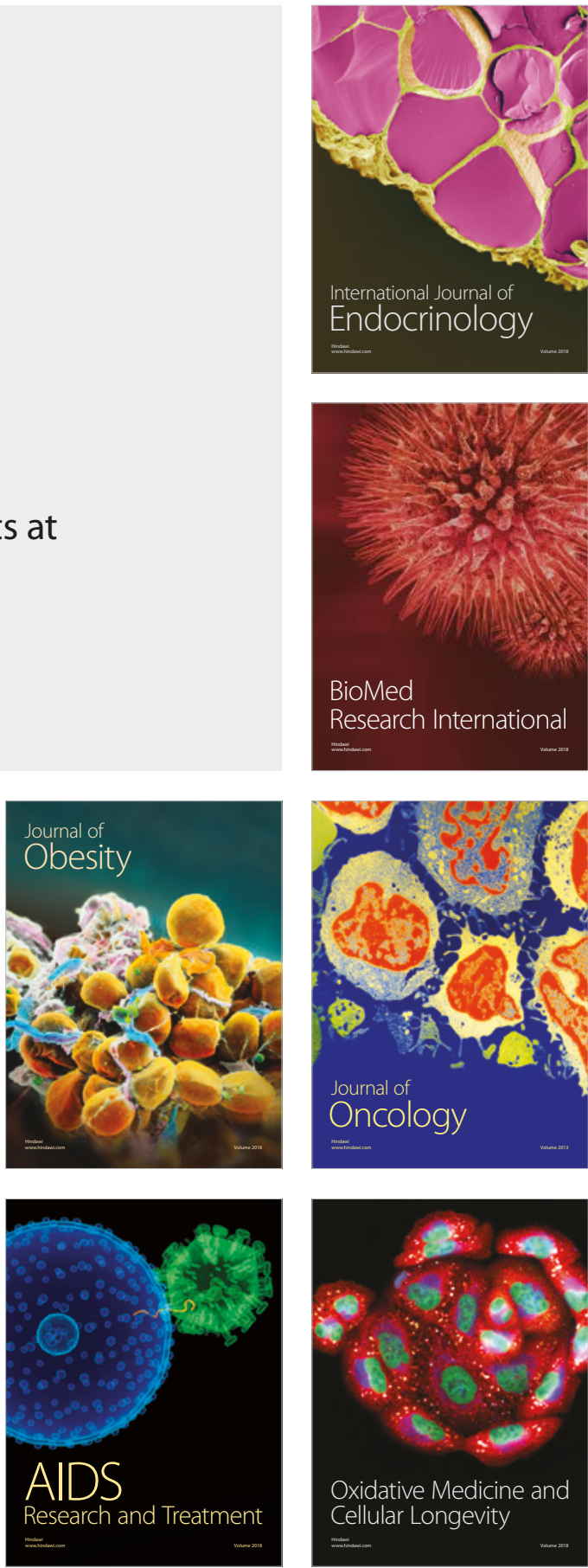